\title{
Flexible Free-standing PEDOT:PSS/MnO 2 Films as Electrode Material for Supercapacitors
}

\author{
Xuejing $\mathrm{Li}^{1,2}$, Chen Zhou ${ }^{3}$, Lanlan Shen ${ }^{3}$,Weiqiang Zhou ${ }^{1}$, Jingkun Xu ${ }^{1, *}$, Chan Luo ${ }^{3}$, Jian Hou ${ }^{2}$, \\ Rongri Tan ${ }^{3, *}$ and Fengxing Jiang ${ }^{3, *}$ \\ ${ }^{1}$ College of Pharmacy, Jiangxi Science and Technology Normal University, Nanchang 330013, P. R. \\ China \\ ${ }^{2}$ State Key Laboratory for Marine Corrosion and Protection, Luoyang Ship Material Research \\ Institute, Qingdao 266101, P. R. China \\ ${ }^{3}$ Jiangxi Key Laboratory of functional organic molecules, Jiangxi Science and Technology Normal \\ University, Nanchang 330013, P. R. China \\ Corresponding authors. Fax: +86-791-83823320, Tel.: +86-791-88537967 \\ *E-mail: xujingkun1971@yeah.net (J. Xu); rrtan@163.com (R. Tan); f.x.jiang@live.cn (F. Jiang)
}

doi: $10.20964 / 2019.05 .12$

Received: 3 August 2018 / Accepted: 28 September 2018 / Published: 10 April 2019

\begin{abstract}
A free-standing and flexible film is desired for wearable energy devices in the future. $\mathrm{MnO}_{2}$ has attracted wide interest as a promising electrode material for high energy density supercapacitors. However, it suffers from the poor electron transfer and film-forming properties. On the contrary, the excellent conducting polymer poly(3,4-ethylenedioxythiophene):poly(styrene sulfonate) (PEDOT:PSS) has a high electrical conductivity with poor specific capacitance. Herein, we designed and fabricated a composite film by combining PEDOT:PSS and $\mathrm{MnO}_{2}$ nanoparticles (NPs) via a facile dilution-filtration. $\mathrm{MnO}_{2}$ NPs were prepared by a simple and environmental friendly method. PEDOT:PSS plays a significant role on the formation of free-standing and flexible composite film. Meanwhile, it enhances the electron transfer property of $\mathrm{MnO}_{2}$ NPs due to the high electrical conductivity of PEDOT:PSS. The as-prepared PEDOT:PSS/MnO 2 composite films show enhanced electrochemical performance and specific capacitance, which is three times higher than PEDOT:PSS films. This facile composite method provide a potential application in future flexible supercapacitor.
\end{abstract}

Keywords: Free-standing films; flexible supercapacitor; PEDOT:PSS; $\mathrm{MnO}_{2}$

\section{FULL TEXT}

(C) 2019 The Authors. Published by ESG (www.electrochemsci.org). This article is an open access article distributed under the terms and conditions of the Creative Commons Attribution license (http://creativecommons.org/licenses/by/4.0/). 